\title{
ENSEÑAR Y APRENDER BIOLOGÍA EVOLUTIVA EN EL SIGLO XXI
}

\section{TEACHING AND LEARNING EVOLUTIONARY BIOLOGY IN THE $21^{\mathrm{ST}}$ CENTURY}

Recibido: 10-10-2012

Aceptado: 06-12-2012

\section{Por: Rosaura Ruiz Gutiérrez', Eréndira Álvarez Pérez², Ricardo Noguera Solano, Martha Susana Esparza Soria ${ }^{4}$}

Resumen: La biología evolutiva es una disciplina integradora; sin embargo, en los procesos de enseñanza-aprendizaje ha ocupado espacios curriculares exiguos, que se reducen a un tema o inciso de los programas de estudio, lo que resulta insuficiente para enseñar y aprender biología en la formación científica básica, media superior y superior. Ante esa situación, el objetivo de este escrito es plantear que en tanto los fenómenos biológicos se explican desde su funcionalidad e historia evolutiva, es necesario que los contenidos curriculares, la formación docente y los recursos didácticos den cuenta, al mismo tiempo, de la naturaleza histórica y funcional de lo vivo; solo así podrá lograrse la enseñanza y el aprendizaje del pensamiento biológico.

Palabras clave: biología evolutiva, biología funcional, enseñanza, aprendizaje, didáctica.

Abstract: The evolutionary biology is fundamental in the unification of the biological sciences. However, in the teaching-learning processes is reduced to a topic or subsection of programs of study, which is insufficient for teaching and learning biology in the basic scientific training, and higher education. In view of this situation, this paper aims to raise that by reason of as biological phenomena are explained from its functionality and evolutionary history, it is necessary that the curricular content, teacher training and teaching resources realize, at the same time, historical and functional nature of living things; only this way could be achieved the teaching and learning of biological thought.

Key words: evolutionary biology, functional biology, teaching, learning, didactics.

\footnotetext{
${ }^{1}$ Coordinadora del Grupo de Estudios Filosóficos, Históricos y Sociales de la Ciencias del Departamento de Biología Evolutiva, Facultad de Ciencias, Universidad Nacional Autónoma de México. rosaura@ciencias.unam.mx

2 Profesor asociado " $C$ " de tiempo completo del Grupo de Estudios Filosóficos, Históricos y Sociales de la Ciencias del Departamento de Biología Evolutiva, Facultad de Ciencias, Universidad Nacional Autónoma de México.ealvarezperez@yahoo.com.mx

${ }^{3}$ Profesor asociado " $\mathrm{C}$ " de tiempo completo del Grupo de Estudios Filosóficos, Históricos y Sociales de la Ciencias del Departamento de Biología Evolutiva, Facultad de Ciencias, Universidad Nacional Autónoma de México.rns@ciencias.unam.mx

${ }^{4}$ Profesora de la asignatura "Historia y filosofía de la Biología" y del taller "Estudios filosóficos, históricos y sociales de la biología evolutiva", en la carrera de Biología, Facultad de Ciencias, Universidad Nacional Autónoma de México. sesparzas@ciencias.unam.mx
} 


\section{Introducción}

A partir del desarrollo de la teoría evolutiva, en especial del darwinismo y su difusión a nivel mundial, se ha planteado la necesidad de su enseñanza como una disciplina fundamental, no solo para la formación de los nuevos científicos sino como parte de la cultura general de la población. Dada su importancia, pero al mismo tiempo su complejidad, se ha hecho necesario realizar investigaciones acerca de los problemas que surgen respecto a su aprendizaje.

Numerosas investigaciones muestran que existen problemas de diversa índole para enseñar y aprender biología evolutiva, situación generalizada en países de distintas latitudes y en todos los grados escolares (Jiménez Aleixandre, 1991, 2002, 2003, 2005; González Galli et al., 2005; González Galli y Meinardi, 2009, 2011; Geraedts y Boersma, 2006; Kampourakis y Zogza, 2007; Jensen y Moore, 2007, Hernández et al., 2009, entre otros). Dificultades, prohibiciones, limitaciones, resistencias, errores, ausencias, conflictos, controversias, prejuicios, tensiones, obstáculos epistemológicos, son todos términos asociados a la enseñanza de uno de los conocimientos más influyentes en la historia de la humanidad. La relevancia del tema y los problemas asociados a su enseñanza son razones suficientes para reflexionar y plantear propuestas que consideren el origen de los problemas implicados.

Diversos autores han destacado la importancia de tomar el enfoque evolutivo como eje estructurador de la enseñanza de la biología contemporánea (Jacob, 1997; Morin, 1999; Fourez, 1994). Sin embargo, como señala Cook (2009), la evolución no ha sido resaltada en los currículos de ciencias de manera proporcional a la importancia que tiene. El caso de México no está exento de esa situación: los temas evolutivos ocupan espacios curriculares restringidos y aislados, tales como un inciso o un capítulo de los programas de estudio en educación básica, una asignatura obligatoria y algunas optativas en la formación de biólogos, formatos por demás insuficientes para enseñar y aprender una disciplina integradora por excelencia y trascendente desde su origen. Por su parte, los contenidos relacionados con la biología de los organismos comúnmente se presentan sin la historia evolutiva que los explica.

En el ámbito científico no hay duda acerca de la importancia de las teorías evolutivas. En el ámbito educativo, en Estados Unidos, la Academia Nacional de Ciencias, la Asociación Americana para el Avance de las Ciencias y la Asociación Nacional de Profesores de Ciencias, resaltan la importancia de utilizar el conocimiento evolutivo como principio explicativo fundamental de las ciencias de la vida (Araujo, 2011). No obstante, el espacio que ocupa en los programas de estudio no es mayor al de otro tema biológico cualquiera.

En este escrito se plantea que, si como señaló Mayr (1998), todo fenómeno viviente debe estudiarse en virtud de dos tipos diferentes de causas: las causas próximas que, en buena medida, son comunes al orden de lo viviente y al orden físico y químico y nos dicen cómo es que algo ocurre; y las causas últimas o remotas, que son específicas de los fenómenos biológicos y nos dicen por qué es que ocurre, entonces la enseñanza y el aprendizaje de la biología requieren la integración de enfoques evolutivos y funcionales, no como temas o incisos de un temario, aislados unos de otros, como se ha presentado hasta ahora en los planes y los programas de estudio en la educación en todos los grados escolares en México. Se propone integrar los contenidos curriculares considerando de cada fenómeno biológico las explicaciones evolutivas y funcionales correspondientes. 


\section{Alfabetización científica individual y social}

La formación de un ciudadano reflexivo, crítico, participativo, que sea a su vez educable, que sea capaz de entender el mundo en general, supone una cultura científica, independientemente de cualquier aplicación práctica inmediata que esta tenga. $Y$ eso incluye a la biología evolutiva (Caponi, 2009). Este conocimiento permite adquirir habilidades para desenvolverse en la vida cotidiana y en el entorno laboral.

En la dimensión social el conocimiento científico, junto con otros campos culturales como la filosofía, el arte, la tecnología, la historia, posibilita la construcción de sociedades más equitativas, plurales, democráticas, con marcos referenciales que permiten tomar mejores decisiones individuales y colectivas, participar de forma organizada e informada en la definición de políticas científicas y tecnológicas y en la elección de estilos de vida.

Dar cultura científica tiene que ver con la formación del ciudadano, con el individuo que se integra a la vida política, en el sentido más amplio de la palabra. La cultura científica hace posible una mejor relación con sí mismo, con los otros y con el ambiente, y aumenta las posibilidades de tomar mejores decisiones en la esfera privada y en la pública. El papel de la biología es central en la formación científica, como disciplina que estudia los fenómenos naturales que suceden en el individuo, desde el nivel genético, celular, tisular, anatómico, fisiológico, conductual, hasta los procesos que se dan en las poblaciones, las especies, la biósfera, y ofrece explicaciones funcionales e históricas para comprenderlos.

Frente a esta aspiración, educativa en principio, y de implicaciones políticas en tanto la alfabetización científica de la sociedad va más allá del ámbito escolar, resulta útil mostrar algunos datos sobre la percepción social de la ciencia en México, que dan una idea de las tareas por realizar.

En la encuesta 2009 (publicada en 2010) sobre la Percepción Pública de la Ciencia y la Tecnología, que elabora anualmente el Consejo Nacional de Ciencia y Tecnología (Conacyt) y que aplica el Instituto Nacional de Estadística y Geografía (Inegi), se reporta que $83.6 \%$ de los encuestados confían más en la fe que en la ciencia; $57.5 \%$ señalan que: "Debido a sus conocimientos, los investigadores científicos tienen un poder que los hace peligrosos; $94.7 \%$ consideró ciencia a la Medicina, $86.6 \%$ a las Matemáticas, $85.6 \%$ a la Física, $84.6 \%$ a la Biología, 38.6\% a la Astrología y 34\% a la Teología (Conacyt, 2010).

Como muestran estos datos, es muy pobre la percepción social de la ciencia y de la naturaleza del conocimiento científico en la sociedad mexicana. Si bien, como se ha señalado, atender esta situación requiere de diferentes actores e instancias, los profesionales de la biología tienen una responsabilidad central al momento de señalar los conocimientos fundamentales que requiere la alfabetización científica de esta disciplina, que sirvan como criterios de selección para el diseño curricular y definición de los contenidos escolares. Uno de estos conocimientos fundamentales para la alfabetización científica es la biología evolutiva.

El conocimiento evolutivo otorga una estructura de pensamiento donde las diferencias individuales se distinguen y valoran como características inherentes a lo vivo en todas sus formas de organización; en esta lógica, no solo importa la particularidad de cada uno: también el conjunto de individuos de la misma especie tiene un valor relevante, porque contiene el acervo actual e histórico de la población en cuestión. El estudio de la 
evolución biológica cuestiona el pensamiento determinista porque en los procesos evolutivos también interviene el azar. En tanto no se tenga pensamiento evolutivo, hay un gran margen para asumir el mundo y los fenómenos biológicos como estáticos y mágicos. La alfabetización evolutiva formula un escenario en el que el cambio es la constante en la naturaleza.

Crear conciencia de la importancia de la evolución en la alfabetización científica, e impulsar acciones en este sentido, fortalece la percepción social de la ciencia, la participación de la sociedad alfabetizada científicamente en la definición de políticas científicas y tecnológicas, y la asimilación de la humanidad como parte integral de la naturaleza.

\section{Enseñar y aprender biología evolutiva en el siglo XXI}

La explicación científica de evolución por variación y selección natural se ha consolidado como paradigma de la biología contemporánea, porque cuenta con evidencias contundentes de diversas disciplinas para explicar la diversidad de lo vivo, su adaptación al medio y la "ancestría" común. La importancia de este conocimiento radica en que transforma la visión de sí mismo, del mundo, de lo vivo y de lo humano. Es paradigmático también como metodología científica, como forma de argumentación, validación y construcción de teorías y modelos para explicar fenómenos naturales. El pensamiento evolutivo es un conocimiento fundamental y complejo, por lo que es necesario definir qué enseñar en cada grado escolar. El reto consiste en construir una visión evolutiva rigurosa, actualizada, suficiente y factible de ser enseñada por los profesores y comprendida por los alumnos en cada caso.

En el contexto educativo un gran número de estudios realizados en distintos países y grados escolares, con diferentes enfoques, han mostrado que existen diversas dificultades para comprender dicha teoría (Hofmann y Weber, 2003; Jiménez Aleixandre, 1991, 2002, 2003, 2005; Berovoides, 1993; Guillén, 1997; Campos et al., 1999; Sánchez, 2000; Ayuso, 2002; Hernández, 2002; Meinardi y Adúriz Bravo, 2002; González Galli et al., 2005, 2008; González Galli, 2008, 2011; González Galli y Meinardi, 2002;Geraedts y Boersma, 2006; Kampourakis, 2007; Moore y Cotner, 2009; Tindon y Lewontin, 2004; Van Dijk y Reydon, 2010, entre otros). Al analizar y relacionar los estudios previos, se observa que los problemas en la enseñanza y el aprendizaje de la biología evolutiva se deben a diversas causas, entre otras: la complejidad intrínseca de esta área de conocimiento; la falta de precisión conceptual de algunos textos (Cft. Jeffery, 1994); la carencia de una visión histórico-filosófica, aunada a la fragmentación de saberes que prevalece en los procesos de enseñanza y de aprendizaje de la ciencia; la ausencia de un marco contextual para enseñar evolución, diseñado y disponible para los profesores (Cft. Cook, 2009). Todos estos factores dificultan la comprensión de las teorías y los conceptos evolutivos, devienen de diferentes fuentes que se conjugan en la interacción didáctica y forman "núcleos o nudos problemáticos" (Hernández et al., 2009). Esos "nudos problemáticos" pueden estar relacionados -y aún ser origen unos de otros- con los obstáculos de alumnos y profesores, la formación y las trayectorias docentes, la naturaleza de los contenidos, las estrategias didácticas y, lo que es más frecuente, diversas combinaciones de estos factores (Álvarez et al., 2010).

En contraparte, no abundan estudios que den cuenta de las dificultades que entraña enseñar y aprender biología funcional y de los resultados obtenidos en ese campo. Cabe preguntarse si esa forma parcial y fragmentada de la enseñanza de lo vivo y esa atención 
asimétrica de la investigación en didáctica de la biología, puede estar apuntando a un problema que no se ha encarado desde su raíz al desvincular, ignorar, desdibujar en la enseñanza de la biología las causas próximas y las causas remotas de los fenómenos biológicos en las que insistió Mayr.

Para ahondar en este punto, resulta útil retomar uno de los tres obstáculos epistemológicos reportados por González Galli (2011) para el aprendizaje del modelo de evolución por selección natural: el pensamiento teleológico. En la biología del organismo o biología funcional hay una "cierta teleología intra-organísmica" (Caponi, 2009), a la que Mayr $(1961,1974)$ llama teleonomía. Este es un tema de debate en la epistemología de la biología y es motivo de gran confusión en la enseñanza y en el aprendizaje de la evolución biológica. Es difícil pensar que podría salvarse este obstáculo si no se estudia, a la vez, la función, la organización y la historia evolutiva de la estructura, la función, la conducta y las combinaciones de estas en el organismo en cuestión.

Es importante recordar que la estructura, la fisiología y las interacciones de los fenómenos biológicos son resultado de un proceso histórico que en algunos casos se remonta al origen de la vida, hace aproximadamente 3800 millones de años. En consecuencia, enseñar y aprender biología implica seleccionar e integrar conocimientos evolutivos y funcionales y definir cuáles son fundamentales en cada grado escolar.

\section{Argumentando desde la historia y la filosofía de la biología}

Siguiendo a Mayr $(1961,1974)$ cuando distingue causas próximas y causas últimas para explicar los fenómenos biológicos, Caponi (2009) señala que hay dos jerarquías en los objetos de estudio en la biología:

- La jerarquía funcional, que se refiere a la biología del organismo como un sistema funcionalmente integrado (habría que agregar en dicha integración la anatomía y la conducta) que se preserva, funciona, se desarrolla y se reproduce de manera autónoma.

- Y la jerarquía genealógica, que da cuenta de la biología de los linajes, de los procesos poblacionales en secuencias generacionales, en otras palabras, de la dimensión evolutiva de lo vivo.

Si esta distinción ha sido ampliamente aceptada entre los biólogos y los filósofos de la biología, es razonable considerar al enseñar y aprender biología la relevancia de:

- Identificar y conocer los fenómenos que suceden en el organismo y preguntarse cómo cada fenómeno contribuye al funcionamiento, la anatomía y la conducta total de este. En este caso, las disciplinas asociadas son la fisiología, la biología del desarrollo, la biología molecular, la genética, la etología, la autoecología (estudio de la relación organismo-ambiente), entre muchas otras.

- Conocer y comprender la biodiversidad, a partir de la historia de los linajes, de los cambios que han tenido a lo largo del tiempo y las relaciones de parentesco entre estos. Cómo se originan los seres vivos, cómo se diversifican y por qué pueden agruparse en tres dominios, cinco reinos y el resto de los grupos taxonómicos; qué características comparten y cuáles permiten distinguir a cada grupo. El origen e historia de procesos como la fotosíntesis, el ciclo de Krebs, la reproducción sexual, la endosimbiosis; cómo se han modificado las funciones vitales en diferentes ambientes; cómo las relaciones bióticas y abióticas alteran la supervivencia y la reproducción y, por lo tanto, los valores demográficos: cómo 
surgen y cómo se extinguen las especies, entre muchos otros fenómenos biológicos. Este es el campo de la biología evolutiva (biogeografía, taxonomía, ecología, paleontología, genética de poblaciones, sistemática, entre otras disciplinas). Y en estas disciplinas tiene un papel relevante el modelo de evolución por variación y selección natural.

En Kansas, en 1999, Gould, para oponerse a la decisión de la junta estatal de educación de prohibir la enseñanza de la evolución, señaló que: "Enseñar biología sin mencionar la evolución es como enseñar inglés sin mencionar la gramática" (citado en Caponi, 2001). A esto puede agregarse que para enseñar biología evolutiva es necesario enseñar biología funcional, ya que la evolución biológica no tiene lugar en el vacío.

Dado que el pensamiento evolutivo implica un cambio de marco referencial en el entendimiento de lo vivo y de la metodología para estudiarlo, es altamente improbable que puedan comprenderse los procesos evolutivos mientras los contenidos, la enseñanza y el aprendizaje de la biología sigan siendo -como los describe Lerner- una lista de hechos inconexos, confusos, sofocantes y en muchos casos contraintuitivos, que requieren una dolorosa memorización (Lerner, 2000). La propuesta es que los ejes estructuradores del currículo sean los fenómenos biológicos, las unidades fundamentales de la vida (herencia, variación, diversidad, adaptación, reproducción, metabolismo, gen, célula, individuo, población, especie, entre otros) y los modelos funcionales y evolutivos que los explican. Son necesarios sistemas curriculares, formación docente y recursos didácticos que integren ambos enfoques y los relacionen con problemas actuales como vacunas, antibióticos, pesticidas, herbicidas, control de plagas, entre otros. De tal forma que los conocimientos sobre biología evolutiva se utilicen para resolver problemas cotidianos.

Si en la historia de la ciencia y del pensamiento en general, la explicación de la evolución por medio de selección natural y variación revolucionó la concepción que se tenía del mundo natural, dio una nueva dimensión de tiempo, provocó una ruptura con el esquema del mundo que hasta entonces prevalecía, y permitió conectar y consolidar distintas disciplinas incipientes del siglo XIX, entonces es razonable pensar que la comprensión de la evolución es estructurante en el desarrollo intelectual, porque permite entender cabalmente los conceptos evolutivos, cimentar una visión dinámica de procesos complejos en la naturaleza e integrar a la especie humana como parte de estos y como factor de cambio, a la vez que promueve valores de respeto hacia la vida como la individualidad, la pluralidad poblacional y la diversidad natural y cultural.

\section{Conclusiones}

La tesis que se ha sostenido en este escrito es que, dada la naturaleza de las preguntas que se plantea la biología, con intenciones didácticas, tendrían que definirse, seleccionarse e integrarse para cada grado escolar los fenómenos biológicos y los modelos funcionales y evolutivos que los explican. En esta lógica, para definir los ejes estructuradores del currículo, desde educación básica hasta educación superior, tendría que partirse de los hechos biológicos y las unidades concretas en las que estos suceden. En consecuencia, la biología evolutiva no será un inciso o un capítulo más de los programas de estudio, sino la explicación necesaria para entender el origen y la historia de los fenómenos biológicos que estudia la biología funcional. 
La enseñanza y el aprendizaje de la biología evolutiva en el siglo XXI, enfrentan el reto de diseñar sistemas curriculares que permitan comprender las causas próximas que explican cómo los seres vivos funcionan, se constituyen, se reproducen, se comportan, y por qué funcionan como lo hacen y tienen la forma que efectivamente tienen. Esto es válido tanto en el diseño curricular como en la formación de profesores, en la generación de recursos didácticos y en la evaluación de los aprendizajes.

La vinculación de los conocimientos biológicos funcionales y evolutivos con problemas contemporáneos es también una tarea pendiente, que contribuirá a la formación de ciudadanos que saben y pueden tomar decisiones informadas y participar en la construcción de mejores relaciones con sí mismos, con la sociedad y con el ambiente.

Las propuestas aquí planteadas sólo podrían llegar a buen puerto si la formación docente se guía por los principios señalados. Para realizar esta labor son necesarios todos los esfuerzos organizados de que sean capaces profesores, científicos, diseñadores curriculares e investigadores en didáctica de la biología.

De igual manera, el diseño de recursos didácticos coherentes con los planteamientos señalados es crucial para viabilizar esta o cualquier otra propuesta. La trascendencia de los procesos educativos está en la claridad de los fines que se persiguen, la interacción y la coherencia de los actores y de las herramientas de que estos dispongan para hacer andar los procesos de enseñanza y de aprendizaje de la biología y de las explicaciones próximas y remotas que esta disciplina ofrece para comprendernos, para vivir en sociedad y para armonizarnos con el medio ambiente.

\section{Referencias}

Álvarez, E., Meinardi, E. y González Galli, L. (2010, octubre). Zonas polémicas de la biología evolutiva y su expresión en la didáctica. Memorias IX Jornadas Nacionales IV Congreso Internacional de Enseñanza de la Biología. San Miguel de Tucumán, Argentina.

Araujo, R. y Roa, R. (2011). Enseñanza de la evolución biológica. Una mirada al estado del conocimiento. Bio-grafía: escritos sobra la Biología y su enseñanza, 4(7), 15-35.

Ayuso, G, y Banet, E. (2002). Pienso más como Lamarck que como Darwin: comprender la herencia biológica para entender la evolución. Alambique. Didáctica de las ciencias experimentales, 32, 39-47.

Berovides, A.V. (1993). Problemas en la enseñanza de la teoría evolutiva en la educación superior. Revista Biología. Facultad de Biología, Universidad de La Habana, 7(2-3), 7987.

Campos, M.A., Sánchez C.A. Gaspar, S. y Paz, V. (1999). La organización conceptual de los alumnos de sexto grado de educación básica acerca del concepto de evolución. Revista Intercontinental de Psicología y Educación, 1 (1 y 2), 39-55.

Caponi, G. (2001). Biología funcional vs biología evolutiva [Versión electrónica]. Episteme, 12, 3-46. 
Caponi, G. (2009). Entrevista al doctor Gustavo Caponi. Revista Bio-grafia: escritos sobre la Biología y su enseñanza, 2(1) ISSN 2027-1034.

Conact (2010). Informe General del Estado de la Ciencia y la Tecnología. México.

Cook, K.A. (2009). Suggested proyect-based Evolution unit for High School: Teaching Content Through Application. The American Biology Teacher, 71(2), 95.

Fourez, G. (1994). Alfabetización científica y tecnológica. Argentina: Ediciones Colihue.

Geraedts Caspar, L. \& Boersma Kerst, T. (2006). Reinventing Natural Selection. International Journal of Science Education, 28(8), 843-870.

Guillén, F. (1997). Construcción de un modelo de enseñanza para biología. Disertación doctoral no publicada, Universidad Nacional Autónoma de México, Ciudad Universitaria, Distrito Federal, México.

González Galli., L., Revel Chion, A. y Meinardi, E. (2008). Actividades centradas en obstáculos para enseñar el modelo de evolución por selección natural. Revista de Educación en Biología, 11(1), 52-55.

González Galli, L. y Meinardi, E. (2009). El pensamiento finalista como obstáculo epistemológico para la enseñanza del modelo darwiniano. Enseñanza de las ciencias. Número extra VIII Congreso Internacional sobre Investigación en Didáctica de las Ciencias, 1274-1276.

González Galli, L., Adúriz-Bravo, A. y Meinardi, E. (2005). El modelo cognitivo de ciencia y los obstáculos epistemológicos en el aprendizaje de la evolución biológica. Enseñanza de la ciencia, Número extra.

González Galli, L. (2011). Obstáculos en el aprendizaje del modelo de evolución por selección natural. Disertación doctoral no publicada, Universidad de Buenos Aires, Argentina.

Hernández R., M.C. (2002). La historia en la enseñanza de la teoría de la selección natural. Disertación doctoral no publicada, Universidad Nacional Autónoma de México, Ciudad Universitaria, Distrito Federal, México.

Hernández R., M.C.. Álvarez, P.E. y Ruiz, G.R. (2009). La selección natural: aprendizaje de un paradigma. Teorema, XXVIII(2), 107-121.

Hofmann, J.R. Weber, B.H. (2003). The Fact of Evolution: Implications for Science Education. Science and Education, 12, 729-760.

Jeffery, K. (1994). A Study of the Presence of Evolutionary Protoconcepts in Pre-High School Texbooks. Journal of Reserch in Science Teaching, 31(5), 507-518.

Jacob, F. (1997). El juego de lo posible. Barcelona: Ediciones Grijalbo Mondadori.

Jiménez Aleixandre, M.P. (1991). Cambiando las ideas sobre el cambio biológico. Enseñanza de las Ciencias, 9(3), 248-256.

Jiménez Aleixandre, M.P. (2002). Aplicar la idea de cambio biológico: ¿por qué hemos perdido el olfato? Alambique. Didáctica de las ciencias experimentales, 32, 48-55. 
Jiménez Aleixandre, M.P. (oord.). (2003). Enseñar ciencias. Barcelona: Editorial GRAÓ.

Jiménez, T. (2009). Los conceptos de población y de especie en la enseñanza de la biología: concepciones, dificultades y perspectivas. [Versión electrónica]. Disertación doctoral no publicada, Universidad de Granada, Granada, España.

Kampourakis, K. (2007). Students preconceptions about evolution: how accurate is the characterization as 'Lamarckian' when considering the history of evolutionary thought. Science \& Educations, 16, 393-422.

Lerner, L.S. (2000). Good Science, Bad Science: Teaching Evolution in the States. [Versión electrónica]. Extraído el 12 de junio de 2012 de http://www.edexcellence.net/library/lerner/gsbsteits.html

Mayr, E. (1961). Cause and Effect in Biology. Science, 134, 1501-1506.

Mayr, E. (1974). Teleological and Teleonomic: A New Analysis. Boston Studies in the Philosophy of Science, 14, 91-117.

Mayr, E., (1998). Así es la Biología. Madrid: Debate.

Meinardi, E. y Adúriz Bravo, A. (2002). Encuesta sobre la vigencia del pensamiento vitalista en profesores de ciencias naturales. Revista Iberoamericana de Educación -OEI. [Versión electrónica]. http://www.rieoei.org/experiencias28.htm

Moore, R. \& Cotner, S. (2009). Rejecting Darwin: The Occurrence \& Impact of Creationism in High School Biology Classrooms. The American Biology Teacher, Online Publication 71(2), 1-4.

Morin, E. (1999). La cabeza bien puesta. Bases para una reforma educativa. Argentina: Nueva Visión.

Sánchez, C. (2000). La enseñanza de la teoría de la evolución a partir de las concepciones alternativas de los estudiantes. Disertación doctoral no publicada, Universidad Nacional Autónoma de México, Ciudad Universitaria, Distrito Federal, México.

Tindon, R. Lewontin, R.C. (2004). Teaching Evolutionary Biology. Genetics and Molecular Biology, 27(1), 124-131.

Van Dijk, E.M. \& Reydon, T. (2010). A Conceptual Analysis of Evolutionary Theory for Teacher Education. Science and Education, 19, 655-677. 